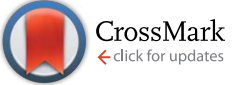

Cite this: RSC Adv., 2017, 7, 5541

\title{
Metal organic frameworks with uni-, di-, and tri- nuclear Cd(II) SBU prepared from 1,3-bis(4-pyridyl) propane and different dicarboxylate ligands: syntheses, structures and luminescent properties $\uparrow$
}

\begin{abstract}
Lei Wu, ${ }^{*}$ Dongdong Chigan, Luke Yan and Huaxin Chen
Three new metal organic frameworks (MOFs) derived from the flexible dipyridyl ligand 1,3-bis(4pyridyl)propane (BPP), $\mathrm{Cd}(\mathrm{BPP})(\mathrm{OBA})\left(\mathrm{H}_{2} \mathrm{O}\right) \cdot(\mathrm{DMF})\left(\mathrm{H}_{2} \mathrm{O}\right)$ (1), $\mathrm{Cd}(\mathrm{BPP})(4-\mathrm{HOIP}) \cdot(\mathrm{DMF})_{0.5}\left(\mathrm{H}_{2} \mathrm{O}\right)$ (2), $\left[\mathrm{Cd}_{6}(\mathrm{BPP})(\mathrm{BPDC})_{7}(\mathrm{DMF})_{2}\right]^{2-} \cdot\left(\mathrm{H}_{3} \mathrm{O}^{+}\right)_{2}\left(\mathrm{H}_{2} \mathrm{O}\right)_{7}(3),\left(\mathrm{H}_{2} \mathrm{OBA}=4,4^{\prime}\right.$-oxybis(benzoic acid), 4- $\mathrm{H}_{3} \mathrm{OIP}=$ 4-hydroxyisophthalic acid, $\mathrm{H}_{2} \mathrm{BPDC}=4,4^{\prime}$-biphenyldicarboxylic acid, DMF $=\mathrm{N}, \mathrm{N}$-dimethylformamide) have been synthesized under mild conditions. By changing the co-dicarboxylate ligands, the three structures are based on uni-, di-, and tri-nuclear Cd(॥) SBUs (secondary building units). Notably, compound 3 has an unusual 3D pillared framework with 2-fold interpenetrated hex topology. Most interestingly, in compound 3, BPP and BPDC ligands play the roles of two kinds of pillars, and support the same 2D layers alternatively leading to a special anionic 3D framework. To the best of our knowledge, such an arrangement has not been reported to date. Moreover, the three compounds exhibit strong luminescent emission at room temperature in the solid state.
\end{abstract}

Received 15th November 2016

DOI: $10.1039 / c 6 r a 26855 b$

www.rsc.org/advances

\section{Introduction}

The rational design and synthesis of MOFs constitutes a current prosperous research field that is attracting intensive attention owing to the scientific interest in the creation of intriguing aesthetic topological structures, as well as their potential applications in gas storage, separation, catalysis, and magnetic and optical devices. ${ }^{\mathbf{1 - 4}}$ It has been realized that the self-assembly of MOFs is influenced by many subtle factors, such as the $\mathrm{pH}$ of the solution, the reaction temperature and time, the nature of the metal ions and counter ions, the coordination modes of the organic ligands, and so on. ${ }^{5-9}$ Therefore, great efforts have been dedicated to exploring the assembly principle of MOFs. As it is known, different donor groups can hold great promise for incorporation of accessibility, structural diversity and geometrical control of properties into the resulting networks. A lot of research groups have been particularly interested in frameworks with $\mathrm{N}$-donor and O-donor mixed ligands systems, which show extensive functional properties and dynamic features. ${ }^{10-13}$ However, the influence of the ligands on the resulting networks in flexible $\mathrm{N}$-donor and O-donor mixed ligands systems has not been well

Institute of Polymer Materials, School of Materials Science and Engineering, Chang'an University, Xi'an 710064, China. E-mail:wulei@chd.edu.cn

$\dagger$ Electronic supplementary information (ESI) available: Crystallographic information files (CIF), PXRD patterns, TGA curves, the selected bond lengths and angles as well as the extensive figures. CCDC 756103-756105. For ESI and crystallographic data in CIF or other electronic format see DOI: 10.1039/c6ra26855b investigated. Thereby, we have embarked on a project to investigate the influence of the ligands on the diversity in assembly of the resulting structures from the $\mathrm{N}$-donor and O-donor mixed ligands systems.

During the last two decades, more and more MOFs have been reported with various topologies. Among the diverse structures, the four-connected nets are the most popular according to the reviews by O'Keeffe, Yaghi and co-workers, such as dia, nbo, pts or cds topologies, which have been widely reported..$^{\mathbf{1 4}-18}$ However, MOFs based on five-connected or eight-connected nets, such as hex-type nets, are comparatively rare. ${ }^{19,20}$ Therefore, herein we hope to get more new types of structures when employing common organic linkers.

Taking into account all the aforementioned topics, we employed BPP as a common and flexible organic ligand into our synthetic strategy. The BPP ligand can give rise to $T T, T G, G G$ and $G G^{\prime}$ conformations ( $T=$ trans, $G=$ gauche), thanks to the free twist of the longer intermediate chain (propyl group: ${ }^{-} \mathrm{CH}_{2}-$ $\mathrm{CH}_{2}-\mathrm{CH}_{2}-$ ). Thereby it can adopt different conformations to take part in the coordination of the metal, resulting in a large variety of network topologies. ${ }^{2-25}$ For the mixed O-donor ligands, we employed $\mathrm{H}_{2} \mathrm{OBA}$, 4- $\mathrm{H}_{3} \mathrm{OIP}$, and $\mathrm{H}_{2}$ BPDC. 4- $\mathrm{H}_{3} \mathrm{OIP}$ and $\mathrm{H}_{2} \mathrm{OBA}$ exhibit a $\mathrm{V}$-shape architecture with similar angles (around $120^{\circ}$ ) built between the two carboxyl groups towards the core of the ligand. However, a main difference appears in terms of flexibility since $4-\mathrm{H}_{3} \mathrm{OIP}$ is rigid while $\mathrm{H}_{2} \mathrm{OBA}$ can bend or rotate freely owing to the central $\mathrm{O}$ atom (ether group). $\mathrm{H}_{2}$ BPDC is commonly considered as a long and rigid linear 
ligand because it is unable to bend and the angle of the two aromatic carboxyl groups towards the centre of the ligand is $180^{\circ}$. With the systematic variation of the O-donor ligands, three new Cd(II) MOFs have been synthesized under mild conditions. Compound $\mathbf{1}$ features a 2D four-connected sql network with uni-nuclear Cd(II) SBUs, whereas compound 2 possesses a 3D distorted cds architecture with di-nuclear $\mathrm{Cd}(\mathrm{II})$ SBUs and compound 3 has a rare 2-fold interpenetrated 3D pillared hex framework with tri-nuclear Cd(II) SBUs. Their frameworks display outstanding structural diversification owing to the variation of the dicarboxylate ligands. Unexpectedly, we found an unusual phenomenon linked to compound 3 in which there exist two kinds of pillars that are arranged alternately in the same layer to construct an atypical anionic 3D framework. To the best of our knowledge, it is the first MOF reported to date with such a feature. Herein, we describe the three structures and discuss the influence of the nature of the ligands on the remaining topologies. The thermal stability and the luminescent properties are also investigated.

\section{Experimental}

\subsection{Materials and measurements}

All chemicals purchased were of reagent grade or better and were used without any further purification. The elemental analyses for $\mathrm{C}, \mathrm{H}$, and $\mathrm{N}$ and the inductively coupled plasma (ICP) analysis were carried out on a PerkinElmer 240C elemental analyzer and PerkinElmer Optima 3300 DV ICP spectrometer, respectively. The infrared (IR) spectra were recorded (400-4000 $\mathrm{cm}^{-1}$ region) on a Nicolet Impact 410 FTIR spectrometer by using the $\mathrm{KBr}$ pellets method. Powder X-ray diffraction (PXRD) data were collected on a Siemens D5005 diffractometer with $\mathrm{Cu} \mathrm{K} \alpha$ radiation $(\lambda=1.5418 \AA$ ). TGA (thermogravimetric analyses) were performed under nitrogen with a heating rate of $5{ }^{\circ} \mathrm{C} \min ^{-1}$ using a PerkinElmer TGA 7 thermogravimetric analyzer. Luminescence spectroscopy data were recorded on an LS55 luminescence spectrometer.

\subsection{Synthesis of compounds}

Compound 1. $\mathrm{Cd}\left(\mathrm{NO}_{3}\right)_{2} \cdot 4 \mathrm{H}_{2} \mathrm{O}(0.06 \mathrm{~g}, 0.2 \mathrm{mmol})$, BPP ( $0.04 \mathrm{~g}, 0.2 \mathrm{mmol})$, and $\mathrm{H}_{2} \mathrm{OBA}(0.05 \mathrm{~g}, 0.2 \mathrm{mmol})$ were dissolved in DMF $(10.00 \mathrm{~mL}) / \mathrm{H}_{2} \mathrm{O}(1.00 \mathrm{~mL})$ mixture, then a DMF solution $(0.50 \mathrm{~mL})$ containing 2-propanolamine $(0.05 \mathrm{~mL})$ was slowly diffused into the mixture over 3 day at $85{ }^{\circ} \mathrm{C}$. The resulting colorless, block-shaped single crystals of compound 1 were collected by filtration, washed with DMF and dried in air (yield: $70 \%$, based on cadmium). IR: $3332 \mathrm{~m}, 3062 \mathrm{w}, 2944 \mathrm{w}, 2856 \mathrm{w}$, $1668 \mathrm{~s}, 1596 \mathrm{~s}, 1558 \mathrm{~s}, 1500 \mathrm{~s}, 1392 \mathrm{~s}, 1238 \mathrm{~s}, 1159 \mathrm{~s}, 1089 \mathrm{~m}, 1014 \mathrm{~m}$, $875 \mathrm{~m}, 790 \mathrm{~s}, 663 \mathrm{~m}, 516 \mathrm{~m}$. Elemental analysis and ICP calculated for $\mathrm{Cd}(\mathrm{BPP})(\mathrm{OBA})\left(\mathrm{H}_{2} \mathrm{O}\right) \cdot(\mathrm{DMF})\left(\mathrm{H}_{2} \mathrm{O}\right)\left(\mathrm{C}_{30} \mathrm{H}_{33} \mathrm{~N}_{3} \mathrm{O}_{8} \mathrm{Cd}\right)(676.02 \mathrm{~g}$ $\mathrm{mol}^{-1}$ ) (\%): C, 53.30; H, 4.92; N, 6.22; Cd, 16.63; found (\%): C, 53.03; H, 5.09; N, 6.57; Cd, 16.35.

Compound 2. The procedure was similar to that used for compound 1, except that $\mathrm{H}_{2} \mathrm{OBA}(0.05 \mathrm{~g}, 0.2 \mathrm{mmol})$ was replaced by $4-\mathrm{H}_{3} \mathrm{OIP}(0.04 \mathrm{~g}, 0.2 \mathrm{mmol})$. The resulting colorless, blockshaped single crystals were collected by filtration, washed by
DMF and then dried in air (yield: $65 \%$, based on cadmium). IR: 3433 m, 3067w, 2928w, 1931w, 1659s, 1593s, 1504s, 1394s, $1267 \mathrm{w}, 1180 \mathrm{~m}, 1105 \mathrm{~m}, 1063 \mathrm{w}, 1007 \mathrm{~m}, 860 \mathrm{~m}, 771 \mathrm{~s}, 677 \mathrm{~m}$, $526 \mathrm{w}$. Elemental analysis and ICP calculated for $\mathrm{Cd}(\mathrm{BPP})(4-$ HOIP $) \cdot(D M F)_{0.5}\left(\mathrm{H}_{2} \mathrm{O}\right)\left(\mathrm{C}_{22.5} \mathrm{H}_{27.5} \mathrm{~N}_{2.5} \mathrm{O}_{7.5} \mathrm{Cd}\right)\left(563.39 \mathrm{~g} \mathrm{~mol}^{-1}\right)$ (\%): C, 47.96; H, 4.93; N, 6.22; Cd, 19.95; found (\%): C, 48.10; H, 4.79; N, 6.58; Cd, 19.77 .

Compound 3. The procedure was similar to that used for compound 1, except that $\mathrm{H}_{2} \mathrm{OBA}(0.05 \mathrm{~g}, 0.2 \mathrm{mmol})$ was replaced by $\mathrm{H}_{2} \mathrm{BPDC}(0.04 \mathrm{~g}, 0.2 \mathrm{mmol})$. The resulting colorless, rodshaped single crystals were collected by filtration, washed by DMF and dried in air (yield: 55\%, based on cadmium). IR: $3448 \mathrm{w}$, 3064w, 2929w, 1934w, 1660s, 1579s, 1519s, 1394s, 1178m, 1099m, $1063 \mathrm{w}, 1006 \mathrm{~m}, 854 \mathrm{~m}, 773 \mathrm{~s}, 682 \mathrm{~m}, 523 \mathrm{w}$. Elemental analysis and ICP calculated for $\left[\mathrm{Cd}_{6}(\mathrm{BPP})(\mathrm{BPDC})_{7}(\mathrm{DMF})_{2}\right]^{2-} \cdot\left(\mathrm{H}_{3} \mathrm{O}^{+}\right)_{2}\left(\mathrm{H}_{2} \mathrm{O}\right)_{7}$ $\left(\mathrm{C}_{117} \mathrm{H}_{104} \mathrm{~N}_{4} \mathrm{O}_{39} \mathrm{Cd}_{6}\right)\left(2864.58 \mathrm{~g} \mathrm{~mol}^{-1}\right)(\%): \mathrm{C}, 49.06 ; \mathrm{H}, 3.66 ; \mathrm{N}$, 1.96; Cd, 23.54; found (\%):C, 49.38; H, 3,87; N, 1.79; Cd, 23.69.

\subsection{X-ray crystallographic study}

Diffraction intensities for compound 1, compound 2 and compound 3 were collected on a computer-controlled Bruker SMART CCD diffractometer equipped with graphite-mono chromated Mo $\mathrm{K} \alpha(\lambda=0.71073 \AA)$ radiation at room temperature by using the $\omega$-scan technique. Raw data for all structures were processed using SAINT-5.0 and absorption corrections were applied using SADABS. ${ }^{26,27}$ These structures were solved by direct methods. All non-hydrogen atoms were refined anisotropically. Hydrogen atoms were fixed at calculated positions and refined by using a riding mode. All calculations were performed using the SHELXTL-97 program. $^{28}$ The main crystallographic data and structure refinement parameters are summarized in Table 1.

Table 1 Crystallographic data and structure refinement for compound 1, compound 2 and compound 3

\begin{tabular}{|c|c|c|c|}
\hline & 1 & 2 & 3 \\
\hline $\begin{array}{l}\text { Empirical } \\
\text { formula }\end{array}$ & $\mathrm{C}_{30} \mathrm{H}_{29} \mathrm{CdN}_{3} \mathrm{O}_{7}$ & $\mathrm{C}_{21} \mathrm{H}_{18} \mathrm{CdN}_{2} \mathrm{O}_{5} \mathrm{O}_{5}$ & $\mathrm{C}_{117} \mathrm{H}_{86} \mathrm{Cd}_{6} \mathrm{~N}_{4} \mathrm{O}_{31}$ \\
\hline$F_{\mathrm{w}}$ & 655.96 & 490.78 & 2718.36 \\
\hline$T(\mathrm{~K})$ & $293(2)$ & $293(2)$ & $293(2)$ \\
\hline$\lambda(\AA)$ & 0.71073 & 0.71073 & 0.71073 \\
\hline Crystal syst. & Monoclinic & Monoclinic & Monoclinic \\
\hline Space group & $P 2_{1} / c($ no. 14$)$ & $C 2 / c($ no. 15$)$ & $C 2 / c$ (no. 15$)$ \\
\hline$a(\AA)$ & $15.3271(3)$ & $16.375(3)$ & $53.600(11)$ \\
\hline$b(\AA)$ & $17.5154(6)$ & $12.766(3)$ & $13.976(3)$ \\
\hline$c(\AA)$ & $11.6216(3)$ & $25.320(5)$ & $47.389(9)$ \\
\hline$\alpha\left(^{\circ}\right)$ & 90.00 & 90.00 & 90.00 \\
\hline$\beta\left(^{\circ}\right)$ & $101.145(2)$ & $98.43(3)$ & $118.27(3)$ \\
\hline$\gamma\left({ }^{\circ}\right)$ & 90.00 & 90.00 & 90.00 \\
\hline$V\left(\AA^{3}\right)$ & $3061.10(14)$ & $5235.8(19)$ & $31266(14)$ \\
\hline$Z$ & 4 & 8 & 8 \\
\hline$\rho_{\text {calc. }}\left(\mathrm{Mg} \mathrm{m}^{-1}\right)$ & 1.423 & 1.245 & 1.155 \\
\hline$\mu\left(\mathrm{mm}^{-1}\right)$ & 0.762 & 0.861 & 0.859 \\
\hline GOF on $F^{2}$ & 0.958 & 1.225 & 1.086 \\
\hline$R^{a}[I>2 \delta(I)]$ & 0.0545 & 0.0668 & 0.0873 \\
\hline$R_{\mathrm{w}}^{b}$ & 0.1321 & 0.2401 & 0.2798 \\
\hline
\end{tabular}


CCDC-756103 (1), CCDC-756104 (2) and CCDC-756105 (3) contain the supplementary crystallographic data for this paper. $\dagger$

\section{Results and discussion}

\subsection{Description of the structures}

Structure of compound 1. Single crystal X-ray diffraction analysis reveals that compound $\mathbf{1}$ crystallizes in the monoclinic system, space group $P 2_{1} / c$ (no. 14). As shown in Fig. 1a, the uninuclear Cd(II) SBU consists of a crystallographic hexacoordinated $\mathrm{Cd}(\mathrm{II})$. The coordination involves four oxygen from one chelating bidentate and one monodentate carboxyl groups of two OBA (Cd-O of 2.224(3)-2.460(3) А), and two nitrogen donors from two BPP ligands (Cd-N of 2.289(3) A and 2.371(4) $\AA$ ). The uni-nuclear Cd(II) SBUs are connected to each other along the crystallographic $a$ axis by the carboxylate ligands to form 1D zigzag chains (Fig. 1b). Then the chains are further linked to the adjacent ones by the flexible BPP ligands along the crystallographic $c$ axis, affording a $2 \mathrm{D}(4,4)$ network with square meshes of $6.2 \times 10.2 \AA^{2}$ (dimensions based on van der Waals' radius of carbon) on the ac plane (Fig. 1c). Each undulated layer interacts with successive one to form double $2 \mathrm{D}$ nets, which pack in $\mathrm{ABAB}$ mode along $b$ axis as shown in Fig. 1d. The DMF guest molecules are statistically disposed inside between two double layers via weak H-bond $\left(d_{(\mathrm{H} \cdots \mathrm{A})}=2.54 \AA, d_{\mathrm{D} \cdots \mathrm{A})}=3.327(10) \AA\right)$ (Fig. S1†). ${ }^{29,30}$ Further insight into the structure of compound 1 can be obtained by reducing the $2 \mathrm{D}$ structure to simple nodelinker geometries and associated nets. ${ }^{31-33}$ The uni-nuclear SBU can be defined as a 4-connected node, and the ligands only serve as bridging linkers. According to the simplification principle, compound $\mathbf{1}$ is formed with a uninodal 4-connected 2D net, and its Schläfli symbol is $\left(4^{4}\right)$, which is a typical sql structure, as shown in Fig. 2.

Structure of compound 2. Single crystal X-ray diffraction analysis reveals that compound 2 crystallizes in the monoclinic system, space group $C 2 / c$ (no. 15). As shown in Fig. 3a, the di-
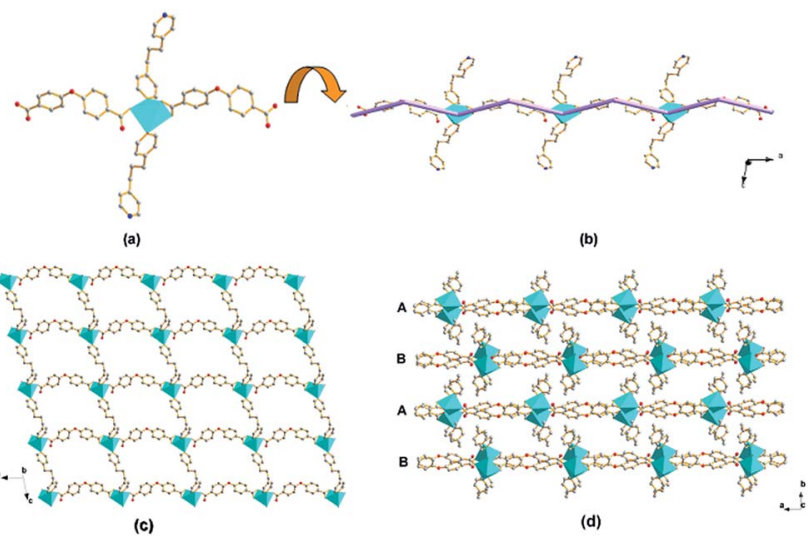

Fig. 1 Schematic illustrations of (a) uni-nuclear Cd(॥) SBU as octahedron; (b) a 1D zig-zag chain with the uni-nuclear Cd(॥) SBUs connected by OBA along a axis; (c) an infinite 2D puckered $(4,4)$ network with the square meshes lay on the ac plane; (d) the double 2D layers stacking in $A B A B$ mode viewed along $c$ axis (color code: $C d$, cyan; $C$, grey; $\mathrm{O}$, red; $\mathrm{N}$, blue).

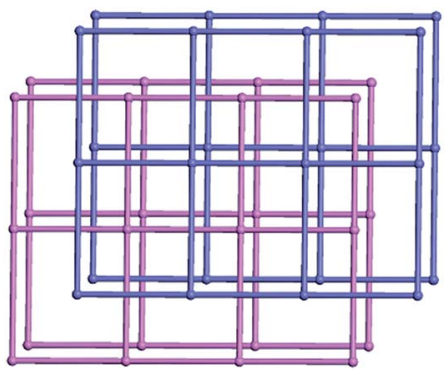

Fig. 2 Schematic illustration of the sql topology of compound 1.
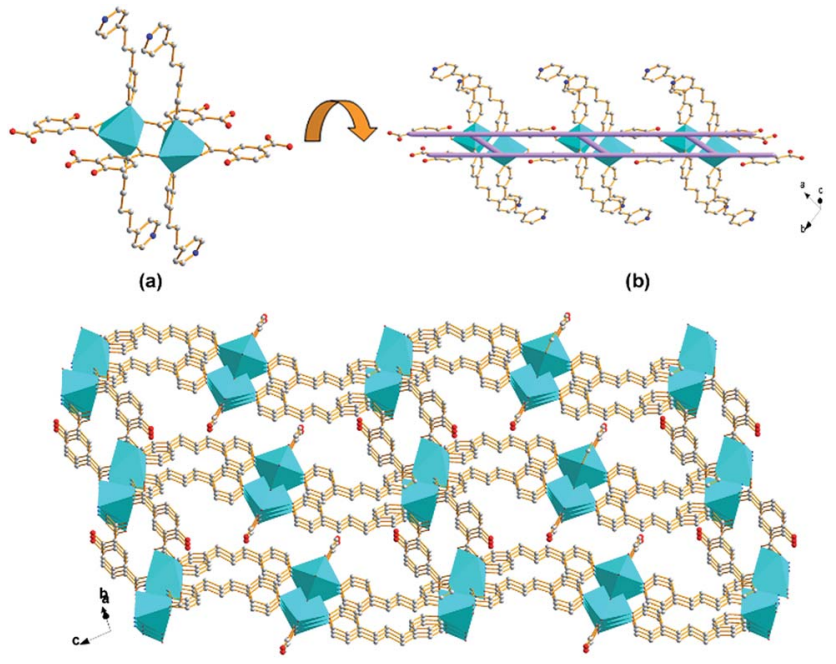

(c)

Fig. 3 Schematic illustrations of (a) di-nuclear Cd(II) SBU; (b) the 1D ladder-like chain with the di-nuclear Cd(॥) SBUs connected by 4-HOIP along the $a$ axis; (c) 3D framework of compound 2 formed by vertical adjacent 1D ladder-like chains connected by the BPP ligands while all of the hydroxyl groups $(-\mathrm{OH})$ remain protonated in the void, viewed along the [110] direction (color code: Cd, cyan; C, grey; O, red; N, blue).

nuclear Cd(II) SBU consists of two hexacoordinated Cd(II) with an identical distorted octahedral coordination environment. Each octahedral coordination mode is completed by four oxygen from one chelating bidentate and two bidentate carboxyl groups borne by three different 4-HOIP (Cd-O of 2.272(6)2.432(7) $\AA$ ), and two nitrogen donors (Cd-N of 2.315(7) and 2.305(7) A). The Cd-Cd separation (center-center distance) is 4.01 $\AA$. As shown in Fig. 3b, the di-nuclear Cd(II) SBUs are linked by 4-HOIP along the $a$ axis to form 1D ladder-like chains. Then two vertical adjacent chains are further connected by the BPP ligands, resulting in a 3D framework with $1 \mathrm{D}$ channels along the [110] direction (Fig. 3c). Notably, the channel is separated by two benzene rings of 4-HOIP into three connected parts while all of the hydroxyl groups $(-\mathrm{OH})$ are free in the void. Two of the parts are triangular channels about $1.6 \times 5.1 \AA^{2}$ (calculated based on van der Waals' radius of carbon), while the other one is almost blocked.

As previously applied for compound 1, a structural simplification based on the di-nuclear Cd(II) SBU as a four-connected 


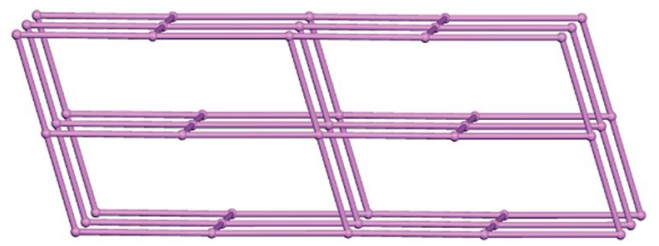

Fig. 4 Schematic illustration of the distorted cds topology of compound 2 .

node, di-BPP and di-4-HOIP in four vertical directions as two types of two-connected linkers, a cds 3D network sustained by 4-coordinated planar nodes is obtained, and its Schläfli symbol is $\left(6^{5} \cdot 8\right)$. Noticeably, owing to the different length of the two types of connections related to two kinds of ligands $(10.382 \AA$ and $14.029 \AA$ ), the distance between two nodes is different. Meanwhile, the torsion of the flexible BPP ligand gives rise to the distortion of the 4-coordinated planar nodes. Consequently, the resulting network of compound 2 is a distorted cds topology (Fig. 4).

Structure of compound 3. The crystallographic analysis reveals that compound 3 crystallizes in the monoclinic system, space group $C 2 / c$ (no. 15). As illustrated in Fig. 5, the tri-nuclear Cd(II) SBU consists of three crystallographic Cd(II) centers. The decahedron coordination of heptacoordinated Cd1 is realized by seven $\mathrm{O}$ donors, which are two pairs of chelating/bridgingbidentate carboxylic $\mathrm{O}$ donors, one bidentate and one pair of chelating bidentate carboxylic $\mathrm{O}$ donors separately from four distinct BPDC. Heptacoordinated $\mathrm{Cd} 3$ is in the same geometry of Cd1, only with one O donor of the coordinated DMF molecule and one $\mathrm{N}$ donor of one BPP ligand taking the place of the couple of chelating carboxylic $\mathrm{O}$ donors on the apical position. However, hexacoordianted $\mathrm{Cd} 2$ adopts the different coordination mode. The octahedral coordination is achieved by four chelating/bridging-bidentate carboxylic $\mathrm{O}$ donors of four distinct BPDC on the equatorial plane and two bidentate carboxylic $\mathrm{O}$ donors of two BPDC occupying the two apical

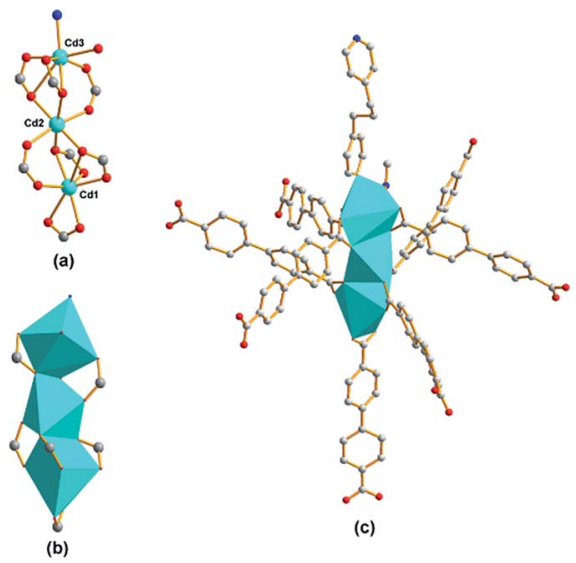

Fig. 5 Schematic illustrations of tri-nuclear Cd(॥) SBU for compound 3 in (a) ball-and-stick mode, (b) polyhedra representation, and (c) SBU coordination mode. coordination sites. The $\mathrm{Cd}-\mathrm{O}$ distances are between 2.189(5) and 2.809(6) $\AA$, and the $\mathrm{Cd}-\mathrm{N}$ distances are between 2.281(6) and 2.285(5) ̊.

The eight vertexes of the tri-nuclear $\mathrm{Cd}(\mathrm{II}) \mathrm{SBU}, \mathrm{Cd}_{3}\left(\mathrm{CO}_{2}\right)_{6} \mathrm{ON}$ cluster (Fig. $5 \mathrm{a}$ and b), on the equatorial plane are linked by six BPDC to form 2D (3, 6)-connected networks along the [10-1] plane (Fig. 6a). The remaining two opposite apical positions are separately occupied by one BPP ligand and one BPDC through the $\mathrm{N}$ donor and a couple of carboxylate $\mathrm{O}$ donors leading to a 3D pillared framework. Notably, the adjacent tri-nuclear Cd(II) SBUs display an inverse orientation along the vertical direction of the $(3,6)$ plane. Therefore the BPDC and BPP ligands occupy the opposite positions compared to the neighboring SBU. Consequently, in compound 3 not only are there two kinds of pillars (BPDC and BPP) but also the distinct pillars alternately support the $2 \mathrm{D}$ planes in the same layer, as shown in Fig. $6 \mathrm{~b}$. To the best of our knowledge, this fantastic arrangement of two pillars in compound 3 is the first reported to date. So unlike most pillared MOFs, compound 3 has an anionic framework with charge-balancing $\mathrm{H}_{3} \mathrm{O}^{+}$in the channels. As shown in Fig. 6c, two identical networks interpenetrate to generate 1D channels of about $4.8 \times 4.8 \AA^{2}$ (calculated based on van der Waals' radius of carbon) along the [101] direction, with coordinated DMF and guest water molecules residing inside.

The topology of compound 3 is studied to further understand the structure. By considering the tri-nuclear SBU as an 8-connected node, and the BPP ligand and BPDC as twoconnected links, an unusual 2-fold interpenetrating 3D pillared hex type network with 8-coordinate nodes is obtained with a Schläfli symbol of $\left(3^{6} \cdot 4^{18} \cdot 5^{3} \cdot 6\right)$, as shown in Fig. 7 .

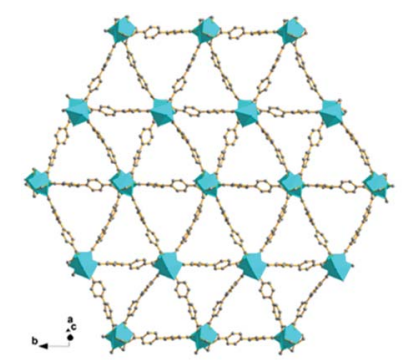

(a)

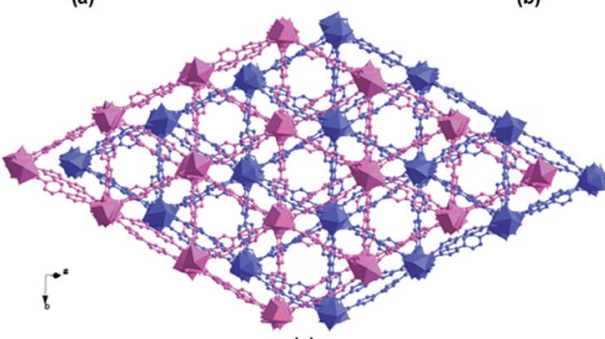

(c)

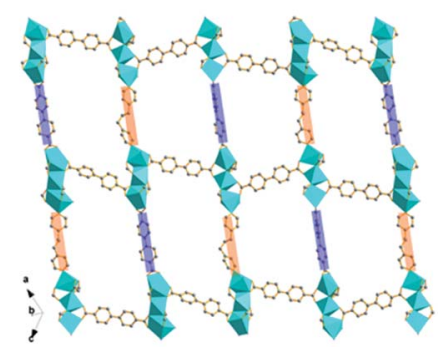

(b)
Fig. 6 Representations of (a) an infinite 2D $(6,3)$ network constructed from tri-nuclear Cd(॥) SBUs connected by BPDC along the [101] plane; (b) 3D framework of compound 3 formed from pillars (BPP and BPDC ligands alternately) supporting the 2D layers, viewed along the [101] direction; (c) 2-fold interpenetrated 3D framework of compound 3 formed by two identical networks interpenetrating, viewed along the [101] direction (color code: Cd, cyan; C, grey; O, red; N, blue). 


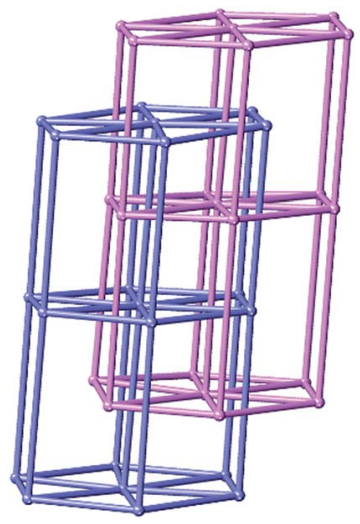

Fig. 7 Schematic illustration of 2-fold interpenetrating hex topology of compound 3 .

Table 2 Comparison of the dicarboxylate ligands in compound 1 , compound 2 and compound 3

\begin{tabular}{|c|c|c|c|c|}
\hline \multirow[b]{2}{*}{ Compound } & \multicolumn{4}{|c|}{ Mixed-dicarboxylate ligands } \\
\hline & Label & Shape & Flexibility & Coordinated mode \\
\hline 1 & OBA & $\mathrm{V}$ & Flexible & $\mu_{2}: \eta^{1}, \eta^{0}, \eta^{1}, \eta^{1}$ \\
\hline 2 & 4-HOIP & V & Rigid & $\mu_{3}: \eta^{1}, \eta^{1}, \eta^{1}, \eta^{1}$ \\
\hline 3 & BPDC & Linear & Rigid & $\begin{array}{l}\mu_{2}: \eta^{1}, \eta^{1}, \eta^{1}, \eta^{1} ; \mu_{3}: \eta^{1}, \eta^{1} \\
\eta^{2}, \eta^{1} ; \mu_{3}: \eta^{1}, \eta^{1}, \eta^{1}, \eta^{1}\end{array}$ \\
\hline
\end{tabular}

\subsection{Effect of the nature of the ligands on the structures}

There is no doubt that the above structure discussion demonstrates the apparent effect of the nature and the configuration of the ligands in the syntheses mixtures on the resulting structures. Although it is impossible to make an exact verdict on the synthesis rule owing to the complexity of the MOFs synthesis system, primitive exploration is still worthwhile for guiding further research. To analyze the structures, a comparison is realized among the different carboxylate ligands and the conformations of the BPP ligand in the three compounds (Tables 2 and 3).

The coordination motifs of the three aromatic dicarboxylate ligands are shown in Scheme 1. The flexibility and the shape of the three ligands as well as the coordination modes of the carboxyl groups are listed in Table 2. Even if OBA and 4-HOIP possess a similar V-shape architecture, the carboxyl groups in the two ligands adopt different coordination modes. The coordination mode of OBA affords $\mu_{2}: \eta^{1}, \eta^{0}, \eta^{1}, \eta^{1}\left(\mu_{n}\right.$ : the bridging

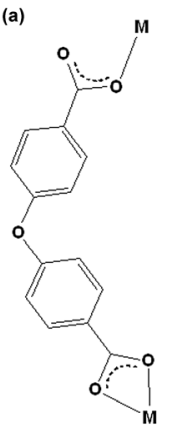

OBA

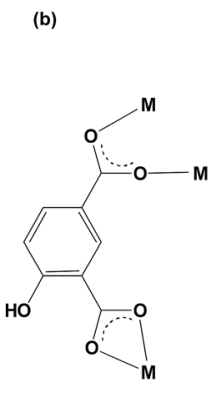

4-HOIP

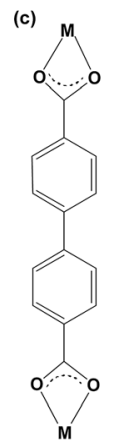

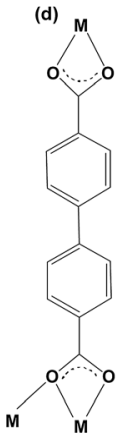

BPDC (e)

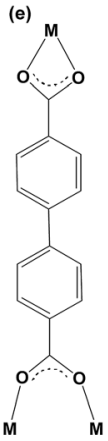

Scheme 1 The coordination modes of the three dicarboxylate ligands in (a) compound 1, (b) compound 2 and (c-e) compound 3 .

mode of the carboxylate ligands; $\eta^{n}$ : the coordination mode of oxygen atoms of carboxyl groups), which includes one chelating-bidentate and one monodentate carboxyl group. Owing to the oxygen atom of the ether group in OBA, the free torsion occurs between the two aromatic carboxyl groups, which leads to one of the carboxyl groups adopting a monodentate mode generating the uni-nuclear SBU. The coordination mode of 4-HOIP affords $\mu_{3}: \eta^{1}, \eta^{1}, \eta^{1}, \eta^{1}$, which includes one chelatingbidentate and one bidentate carboxyl groups. The bidentate carboxyl group of 4-HOIP instead of the corresponding monodentate carboxyl group of OBA leads to di-nuclear SBU. Then the uni- or di-nuclear SBUs are further linked by the carboxylate ligands to form the corresponding zig-zag or ladder-like chains, respectively, in compound 1 and compound 2 (Fig. $1 \mathrm{a}$ and $\mathrm{b}$ and $3 \mathrm{a}$ and $\mathrm{b}$ ). As a comparison, BPDC can be commonly considered as a linear and rigid ligand. Thus, the relatively long distance, the rigidity and the various coordination modes of the carboxyl groups $\left(\mu_{2}: \eta^{1}, \eta^{1}, \eta^{1}, \eta^{1} ; \mu_{3}: \eta^{1}, \eta^{1}, \eta^{2}, \eta^{1} ; \mu_{3}: \eta^{1}, \eta^{1}, \eta^{1}, \eta^{1}\right)$ facilitate the generation of the tri-nuclear $\mathrm{Cd}(\mathrm{II})$ unit, then further form a 2D $(3,6)$ plane along the $[10-1]$ plane (Fig. 5 and 6a).

In addition to the carboxylate ligands, the $\mathrm{N}$-donor ligand also plays a crucial role in the construction of the final framework. Generally, besides the classification of $T / G$ configuration, the conformation of the BPP ligand can be estimated in more details with two factors: (i) the dihedral angle of the two pyridyl rings, named $\phi$; and (ii) the $\mathrm{N}-\mathrm{N}$ distance, defined as $d$. The $T / G$ configurations of all the bidentate BPP ligands in the three compounds are shown in Scheme 2 and Fig. S4 $\dagger$ while $\phi$, $d$ values are listed in Table 3. It is not difficult to find that the BPP ligand adopts the more flexible $T G$ mode with $\phi$ of $65.09^{\circ}$ and $d$ of $8.41 \AA$ in compound 1 . The twisted conformation of

Table 3 Comparison of the configuration of the BPP ligands and frameworks of compound 1, compound 2, and compound 3

\begin{tabular}{|c|c|c|c|c|c|c|}
\hline 2 & $T T$ & 9.99 & 41.28 & Di-Cd & $6^{5} \cdot 8$ & $3 \mathrm{D}$ \\
\hline 3 & $T G$ & 9.65 & 30.99 & Tri-Cd & $3^{6} \cdot 4^{18} \cdot 5^{3} \cdot 6$ & $3 \mathrm{D}$ \\
\hline
\end{tabular}




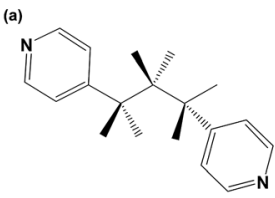

TG

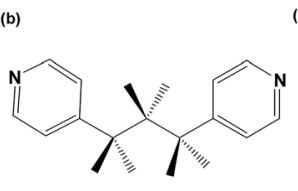

TT

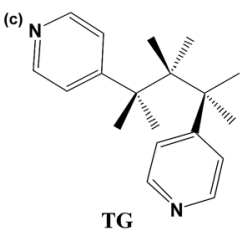

TG
Scheme 2 The T/G conformations of the BPP ligands in (a) compound 1, (b) compound 2, and (c) compound 3.

BPP combined with the monodentate carboxyl group of OBA lead to the tendency to form planar 4-connected nodes. It offers an opportunity to the $\mathrm{O}$ donor of water to occupy one coordination position furnishing the octahedral coordination mode of Cd(II) in uni-nuclear SBU. In compound 2, two $\mathrm{N}$ donors of two parallel BPP ligands occupy the axial position of the di-nuclear Cd(II) SBU. Compared to the flexible conformation of BPP in compound 1, BPP ligands adopt the TT configuration with $\phi$ of $41.26^{\circ}$ and $d$ of $9.99 \AA$, which may be more in favor of interparalleling. Simultaneously, just due to $T T$ configuration and the torsions of the two pyridyls, the two ladder-like chains joined by the same BPP are almost vertical, resulting in a distorted cds topological structure. Although the BPP ligands in compound $\mathbf{3}$ adopt the $T G$ configuration as in compound $\mathbf{1}$, the stretch of the propyl group $\left(-\mathrm{CH}_{2}-\mathrm{CH}_{2}-\mathrm{CH}_{2}-\right)$ makes the BPP ligands longer $(d=9.65 \AA)$, which is approximately equal to the space of the two carboxyl groups of BPDC. Therefore, this makes it possible to generate the two kinds of pillars alternately supporting the $(3,6)$ planes in the same layer to form an 8-connected hexagonal primitive framework.

The three structures in this work clearly reveal that the shape and the flexible abilities of carboxylate ligands, the position and the coordination modes of the carboxyl groups, as well as the configuration of the $\mathrm{N}$-donor ligand, all have a great influence on the target structures. The multi-coordination modes are in favor of the formation of multinuclear SBUs, which are the nodes of the network that profoundly affect the whole topology. Multinuclear SBUs consequently facilitate construct multidimensional frameworks.

\subsection{Thermogravimetric analysis}

The TGA curve of compound 1 exhibits the first weight loss of $14.0 \%$ from 35 to $90{ }^{\circ} \mathrm{C}$ which is attributed to the releasing of free DMF and $\mathrm{H}_{2} \mathrm{O}$ molecules (calc. 13.5\%). The second weight loss of $2.9 \%$ from 90 to $260{ }^{\circ} \mathrm{C}$ corresponds to the dissociation of coordinated $\mathrm{H}_{2} \mathrm{O}$ (calc. $2.6 \%$ ). When the temperature is above $260{ }^{\circ} \mathrm{C}$, compound 1 decomposes and the remaining weight of $19.1 \%$ corresponds to the percentage of $\mathrm{Cd}$ and $\mathrm{O}$ components, CdO (calc. $19.0 \%$ ).

For compound 2, the TGA curve indicates that the first weight loss of $12.9 \%$ from 35 to $135{ }^{\circ} \mathrm{C}$ corresponds to DMF and $\mathrm{H}_{2} \mathrm{O}$ free molecules (calc. $12.9 \%$ ). Then, the structure is stable to $270{ }^{\circ} \mathrm{C}$. When the temperature is above $270{ }^{\circ} \mathrm{C}$, compound 2 decomposes and the remaining weight of $23.2 \%$ corresponds to the percentage of $\mathrm{Cd}$ and $\mathrm{O}$ components, $\mathrm{CdO}$ (calc. $22.9 \%$ ).
For compound 3, the TGA curve indicates that the first weight loss of $5.7 \%$ from 35 to $140{ }^{\circ} \mathrm{C}$ is attributed to decomposition of all free $\mathrm{H}_{2} \mathrm{O}$ molecules (calc. 5.7\%). The second weight loss of $10.1 \%$ from 140 to $260{ }^{\circ} \mathrm{C}$ corresponds to the dissociation of one coordinated DMF molecule and BPP (calc. $9.5 \%$ ). The weight loss of the third step of $11.5 \%$ from 260 to $400{ }^{\circ} \mathrm{C}$ corresponds to the removal of another coordinated DMF molecule and one BPDC (calc. 11.3\%). Above $400{ }^{\circ} \mathrm{C}$, compound 3 decomposes completely, and the remaining weight of $26.9 \%$ is in agreement with the percentage of $\mathrm{Cd}$ and $\mathrm{O}$ components, CdO (calc. 26.9\%).

\subsection{Luminescent properties}

MOFs that contain $\mathrm{d}^{10}$ metal centers are frequently reported to exhibit dramatic luminescent properties, thereby to be promising candidates for photoactive materials with potential applications in chemical sensors, photochemistry, and electroluminescent displays. ${ }^{34-36}$ The solid-state photoluminescence spectra of the compounds have been investigated at room temperature. It can be observed that the intense emission occurred at $396 \mathrm{~nm}, 404 \mathrm{~nm}$ and $369 \mathrm{~nm}$ for compound 1, compound 2 and compound 3 distinctively when the compounds are excited at $330 \mathrm{~nm}, 318 \mathrm{~nm}$ and $308 \mathrm{~nm}$ (Fig. 8b-d). Since $\mathrm{Cd}(\mathrm{II})$ is difficult to oxidize or reduce due to its $\mathrm{d}^{10}$ configuration, the emissions of the compounds are essentially ligand-based, neither metal-to-ligand charge transfer (MLCT) nor ligand-tometal charge transfer (LMCT). ${ }^{\mathbf{4 0}-42}$ To analyze the fluorescence properties of the compounds, the solid-state emission spectra of $\mathrm{H}_{2} \mathrm{OBA}, 4-\mathrm{H}_{3} \mathrm{OIP}, \mathrm{H}_{2} \mathrm{BPDC}$ and the pure BPP ligand were measured. Although the free carboxylate ligands, $\mathrm{H}_{2} \mathrm{OBA}$, 4- $\mathrm{H}_{3} \mathrm{OIP}$ and $\mathrm{H}_{2}$ BPDC can also exhibit fluorescence at room temperature (Fig. S5 $\dagger$ ), the emission resulting from the $\pi^{*} \rightarrow \mathrm{n}$ transition is relatively weak compared to that from the $\pi^{*} \rightarrow \pi$ transition of BPP. With the addition of fluorescence quenching due to the strong electron withdrawing carboxyl group, the
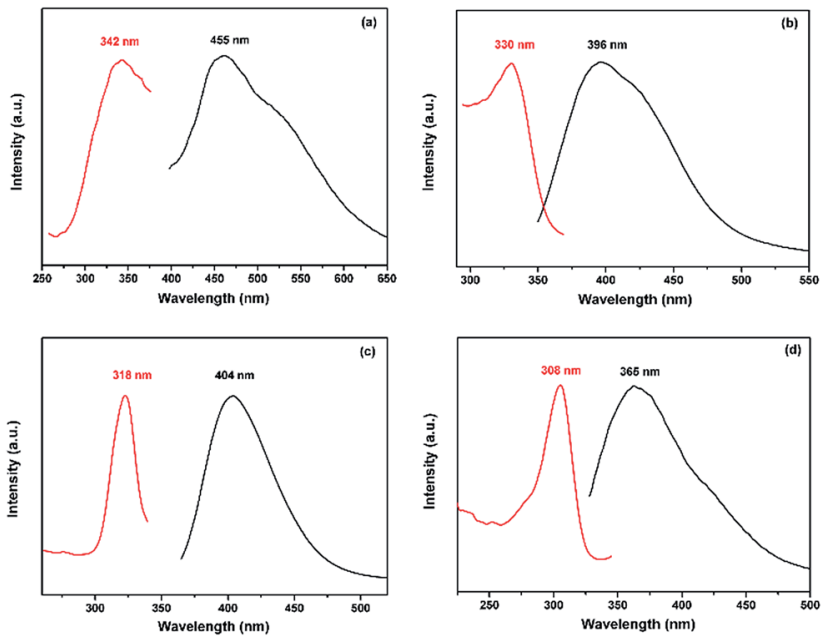

Fig. 8 Solid-state photoluminescence spectra of (a) free BPP, (b) compound 1, (c) compound 2, and (d) compound 3 at room temperature (excitation spectrum, red/left; emission spectrum, black/ right). 
carboxylate ligands almost have no contribution to the fluorescent emission of the compounds. ${ }^{37-39}$ Therefore, the emission band of the compounds may be attributed to the intra-ligand or inter-ligand charge transfer of the BPP ligand. The emission spectra for the compounds exhibit different blue shifts with respect to the emission peak of the free BPP ligand $(455 \mathrm{~nm}$ excited at $345 \mathrm{~nm}$, as shown in Fig. 8a), which may be ascribed to the entirely distinctive coordination environment of the BPP ligand. ${ }^{39,43}$ Furthermore, an obvious blue shift of compound 3 relative to compound $\mathbf{1}$ and compound $\mathbf{2}$ may be due to the degree of $\pi$-overlap of the organic chromophore. The BPP ligands are well separated and have minimal cofacial distances in low-density 3D compound 3 compared to the other two, leading to minimal inter-chromophore interactions, and then a consequence of the obvious blue shift. ${ }^{44}$ Though more detailed theoretical and spectroscopic studies should be performed in order to better understand the luminescent mechanism, the strong fluorescence emissions of those compounds suggest that they may be candidates for potential photoactive applications.

\section{Conclusions}

In summary, we have successfully synthesized three new $\mathrm{Cd}$ (II) MOFs prepared from the flexible ligand BPP in the presence of three distinct aromatic dicarboxylate ligands. The systematic variation of the carboxylate ligands leads to various structures based on uni-, di-, and tri-nuclear SBUs, and from 2D to 3D frameworks. The diverse crystal structures indicate the crucial influence of the organic ligands on the synthesis of the target frameworks. The successful preparation of the three different MOFs provides a meaningful approach for the construction of other coordination polymers with diverse structures under different synthesis conditions. Simultaneously, this work proves again that common organic linkers can be employed to get new types of structures. The successful achievement of compound 3 indicates for the first time that not only can two different kinds of pillars be involved in the same framework, but they can also alternately arrange in the same layer to support the 2D planes, which constructed a special anionic 3D framework. Finally, the strong fluorescence properties of those compounds indicate that they may be suitable as candidates for potential photoactive materials.

\section{Acknowledgements}

The project was supported by the National Natural Science Foundation of China (51502021) and the Special Fund for Basic Scientific Research of Central Colleges, China (2014GB11091).

\section{Notes and references}

1 H. Furukawa, K. E. Cordova, M. O'Keeffe and O. M. Yaghi, Science, 2013, 341, 1230444.

2 D. M. Chen, J. Y. Tian, M. Chen, C. S. Liu and M. Du, ACS Appl. Mater. Interfaces, 2016, 8, 18043.

3 M. Du, C. P. Li, M. Chen, Z. W. Ge, X. Wang, L. Wang and C. S. Liu, J. Am. Chem. Soc., 2014, 136, 10906.
4 D. M. Chen, J. Y. Tian, C. S. Liu and M. Du, Chem. Commun., 2016, 52, 8413.

5 B. S. Zheng, J. F. Bai, J. G. Duan, L. Wojtas and M. J. Zaworotko, J. Am. Chem. Soc., 2011, 133, 748.

6 J. R. Li, J. Sculley and H. C. Zhou, Chem. Rev., 2012, 112, 869.

7 N. Stock and S. Biswas, Chem. Rev., 2012, 112, 933.

8 J. Y. Lee, O. K. Farha, J. Roberts, K. A. Scheidt, S. T. Nguyen and J. T. Hupp, Chem. Soc. Rev., 2009, 38, 1450.

9 Q. Chen, J. B. Lin, W. Xue, M. H. Zeng and X. M. Chen, Inorg. Chem., 2011, 50, 2321.

10 S. Shimomura, N. Yanai, R. Matsuda and S. Kitagawa, Inorg. Chem., 2011, 50, 172.

11 Y. Zhang, T. Wu, R. Liu, T. Dou, X. H. Bu and P. Y. Feng, Cryst. Growth Des., 2010, 10, 2047.

12 Y. J. Cui, Y. F. Yue, G. D. Qian and B. L. Chen, Chem. Rev., 2012, 112, 1126.

13 J. Zhang, W. B. Yang, X. Y. Wu, L. Zhang and C. Z. Lu, Cryst. Growth Des., 2016, 16, 475.

14 M. O'Keeffe, M. Eddaoudi, H. Li, T. Reineke and O. M. Yaghi, J. Solid State Chem., 2000, 152, 1.

15 N. Ockwig, O. D. Friedrichs, M. O'Keeffe and O. M. Yaghi, Acc. Chem. Res., 2005, 38, 176.

16 M. Li, D. Li, M. O'Keeffe and O. M. Yaghi, Chem. Rev., 2014, 114, 1343.

17 C. Wang and W. B. Lin, J. Am. Chem. Soc., 2011, 133, 4232.

18 J. Q. Liu, Y. Y. Wang and Y. S. Huang, CrystEngComm, 2011, 13, 3733.

19 M. Xue, Z. J. Zhang, S. C. Xiang, Z. Jin, C. D. Liang, G. S. Zhu, S. L. Qiu and B. L. Chen, J. Mater. Chem., 2010, 20, 3984.

20 C. Wang and W. B. Lin, J. Am. Chem. Soc., 2011, 133, 4232.

21 H. Q. Hao, J. Wang, W. T. Liu and M. L. Tong, CrystEngComm, 2008, 10, 1454.

22 J. Zhang, E. Chew, S. M. Chen, J. T. H. Pham and X. H. Bu, Inorg. Chem., 2008, 47, 3495.

23 A. J. Hernández-Maldonado, R. R. Arrieta-Pérez, J. N. Primera-Pedrozo and J. Exley, Cryst. Growth Des., 2015, 15, 4123.

24 G. G. Luo, H. B. Xiong and J. C. Dai, Cryst. Growth Des., 2011, 11, 507.

25 L. F. Ma, L. Y. Wang, D. H. Lu, S. R. Batten and J. G. Wang, Cryst. Growth Des., 2009, 9, 1741.

26 SHELXTL, Version 5.1, Siemens Industrial Automation, Inc., 1997.

27 G. M. Sheldrick, SADABS, Program for Empirical Absorption Coordination for Area Detector Data, University of Gottingen, Gottingen, Germany, 1996.

28 G. M. Sheldrick, SHELXTL Version 5.1 Software Reference Manual, Bruker AXS. Inc., Madison, WI, 1997.

29 G. R. Desiraju, Acc. Chem. Res., 1996, 29, 441.

30 J. W. Steed and J. L. Atwood, Supramolecular Chemistry, John Wiley \& Son, Chichester, 2000.

31 A. F. Wells, Further studies of three-dimensional nets, American Crystallographic Association (distributed by Polycrystal Book Service, Pittsburgh, PA), New York, 1979.

32 A. J. Blake, N. R. Champness, P. Hubberstey, W. S. Li, M. A. Withersby and M. Schröder, Coord. Chem. Rev., 1999, 183, 117. 
33 O. D. Friedrichs, M. O'Keeffe and O. M. Yaghi, Acta Crystallogr., Sect. A: Found. Crystallogr., 2003, 59, 22.

34 Q. Wu, M. Esteghamatian, N. X. Hu, Z. Popovic, G. Enright, Y. Tao, M. D'Iorio and S. Wang, Chem. Mater., 2000, 12, 79.

35 J. E. McGarrah, Y. J. Kim, M. Hissler and R. Eisenberg, Inorg. Chem., 2001, 40, 4510.

36 Y. Takashima, V. M. Martínez, S. Furukawa, M. Kondo, S. Shimomura, H. Uehara, M. Nakahama, K. Sugimoto and S. Kitagawa, Nat. Commun., 2011, 2, 168.

37 L. L. Wen, D. B. Dang, C. Y. Duan, Y. Z. Li, Z. F. Tian and Q. J. Meng, Inorg. Chem., 2005, 44, 7161.

38 J. G. Lin, S. Q. Zang, Z. F. Tian, Y. Z. Li, Y. Y. Xu, H. Z. Zhu and Q. J. Meng, CrystEngComm, 2007, 9, 915.

39 C. Ping, Z. Chen, D. L. Gao, B. Zhao, W. Shi and P. Cheng, Cryst. Growth Des., 2010, 10, 4370.
40 X. Shi, G. S. Zhu, Q. R. Fang, G. Wu, G. Tian, R. W. Wang, D. L. Zhang, M. Xue and S. L. Qiu, Eur. J. Inorg. Chem., 2004, 1, 185.

41 X. Shi, G. S. Zhu, X. H. Wang, G. H. Li, Q. R. Fang, X. J. Zhao, G. Wu, G. Tian, M. Xue, R. W. Wang and S. L. Qiu, Cryst. Growth Des., 2005, 5, 341.

42 H. Y. Bai, J. F. Ma, J. Yang, L. P. Zhang, J. C. Ma and Y. Y. Liu, Cryst. Growth Des., 2010, 10, 1946.

43 X. Y. Yu, L. Ye, X. Zhang, X. B. Cui, J. P. Zhang, J. Q. Xu, Q. Hou and T. G. Wang, Dalton Trans., 2010, 39, 10617.

44 C. A. Bauer, T. V. Timofeeva, T. B. Settersten, B. D. Patterson, V. H. Liu, B. A. Simmons and M. D. Allendorf, J. Am. Chem. Soc., 2007, 129, 7136. 